\title{
Cloning, expression and purification of hemagglutinin conserved domain (HA2) of influenza A virus, to be used in broad-spectrum subunit vaccine cocktails
}

\author{
Farahmand $\mathbf{B}^{1^{*}}$, Akbari $\mathrm{A}^{2}$, Akbari $\mathrm{Kh}^{3}$, Fotouhi Chahouki $\mathbf{F}^{1}$, Mehrbod $\mathbf{P}^{1}$, Jalili $\mathbf{N}^{2}$ \\ ${ }^{1}$ Influenza and Other Respiratory Viruses Department, Pasteur Institute of IRAN, Tehran, Iran. \\ ${ }^{2}$ Faculty of Medical sciences, Azad University, Tehran, Iran. \\ ${ }^{3}$ Pharmaceutical Sciences Branch of Islamic Azad University, Tehran, Iran.
}

\begin{abstract}
Introduction: Influenza virus has several conserved peptides which have the capacity to be used as suitable candidates for appropriate and stable vaccine production against different types of influenza viruses. One of these peptides is HA2, the hemagglutinin stalk domain which mediates the membrane fusion and is conserved amongst different sub-types of influenza virus. This peptide is a good candidate for participation in vaccine structure to induce immunity and antibody production. The ensued antibody may hamper the membrane fusion and subsequently the virus propagation. Methods: The peptide sequence of HA2 from influenza virus $\mathrm{A} / \mathrm{Tehran} / 18 / 2010$ (H1N1) was analyzed using in silico tools in order to evaluate its physicochemical properties and identification of its best immunogenic sites. The confirmed sequence was amplified and cloned into a pET28a vector and the recombinant protein was over-expressed prokaryotically and confirmed by Western blotting. Results: The bioinformatics data showed that HA2 peptide stability and immunogenicity. The generated HA2 construct was confirmed by PCR, endonuclease restriction enzyme analysis and sequencing. The expression of HA2 was confirmed by SDS-PAGE and Western blot analysis. The results on the cell lysate demonstrated the high expression of HA2 subunit of the influenza virus hemagglutinin. Conclusion: One of the disadvantages of the current flu vaccines is that they cannot produce efficient broad-spectrum cellular and humoral immune responses against all subtypes of the virus due to the genetic variation of the virus. Therefore, such a conserved protein is potentially a good candidate for production of a broad-spectrum vaccine to prevent influenza epidemics and pandemics.
\end{abstract}

KEYWORDS: influenza virus, hemagglutinin, conserved domain, HA2, vaccine.

\section{INTRODUCTION}

With the onset of the cold season, the incidence of respiratory illnesses such as influenza is increased dramatically which causes mild to severe illnesses and in some cases, death. The causative agent of this disease is a member of the Orthomyxoviridea family which is divided into three types of A, B and C [1]. Type A virus has been accounted for three pandemics of Spain in 1918, Asia in 1957 and Hong Kong in 1968 [1]. The genome of type A virus consist of 8 fragments of RNA which encodes approximately ten proteins $[2,3]$. The two main membrane proteins of the virus are hemagglutinin (HA) and neuraminidase (NA) which are the most important surface glycoproteins and antigens upon which the viral antigenic diversity and the host immunity depend [4].

Due to the biological significance of HA protein of influenza virus, several researches have been conducted on it. This

*Corresponding Author: Behrokh Farahmand, Influenza and Other Respiratory Viruses Department, Pasteur Institute of IRAN, Tehran, Iran.

Email: b farahmand@pasteur.ac.ir

Tel/Fax: (+98) 2166496517 glycoprotein is a trimeric spike and consists of 566 amino acids [5]. A signal peptide sequence at the end of the amine terminal leads the polypeptide towards the endoplasmic network [6]. The HA0 protein is then cleaved into two HA1 (328 amino acids) and HA2 (221 amino acids) subunits by the respiratory tract proteases which remain attached to each other by di-sulfide bonds [7]. The HA1 subunit located at the N-terminal of the molecule includes a spherical head which has cell-receptor binding sites that allow the virus to bind to the sialic acid receptors at the surface of the epithelial cells of the human respiratory tract [8] and possess multiple antigenic regions that can be detected by antibodies [1]. The HA2 subunit is located at the C-terminal of HA glycoprotein. Its primary amino acids (185 residues) are outside of the membrane, 25 amino acids are trans-membrane and 10 amino acids are inside the membrane [9]. The 38 initial primary amino acids located at the $\mathrm{N}$ terminal region are involved in fusion of the virus and the endosomal membranes [7]. As a result of germination and spread of the infection, the humoral and cellular immune responses are stimulated. The secreted antibodies are mainly 
against the HA1 and HA2 antigenic regions [1, 4] which ultimately prevent the virus attachment and replication.

Having a fragmented genome and lack of a proof-reading mechanism for the polymerase enzyme [7], several genetic shifts and drifts occur in two surface glycoproteins (i.e. HA and NA) of the virus. These changes on the new subtype prevent the efficiency of the matched antibodies against the parental subtypes $[10,11]$. These variations cause the viral scape from the host immune system. However, in spite of the sequential changes in HA molecule, there are some epitopes which are conserved in different types of this virus [12, 13]. These conserved epitopes are more in HA2 than HA1 subunit [14]. In fact, the fusion peptide, located in the N-terminal of the HA2 subunit, especially the first 11 amino acids, are conserved among different subtypes of the influenza virus which can be the target of antibodies of the host immune system [15]. Furthermore, the arginine amino acid which binds between HA1 and HA2 and is detected by the host respiratory protease, is also fully conserved [7].

A large number of studies have shown that HA2 subunit has $85 \%$ homology within different subtypes [16]. Regarding the genetic variation of influenza virus which causes annual epidemics, the development of a new vaccine based on the conserved proteins such as HA and M2 have been pursued by the researchers over the last few years. Therefore, in this study, a short segment of an HA subunit (HA2) of influenza A virus/H1N1was isolated and expressed in a prokaryotic host as an appropriate and conserved antigen to be used and assayed in the combination of a new candidates of influenza subunit vaccine in future.

\section{MATERIALS and METHODS}

\section{Bioinformatics analyses}

Firstly, The amino acid sequence of HA from influenza A virus (A/ Tehran/ 18/2010/ H1N1) with the accession code HQ419001.1 was blasted with multiple viral HA2 protein sequences in NCBI (National Center for Biotechnology Information) database [17]. The desired sequence with respect to the purpose of the study which was the expression of a viral protein in the prokaryotic expression system, was investigated using codon optimization software. Then, the optimized amino acid sequence using a software at Expasy database [18] was evaluated for physicochemical properties as well as the immunogenicity and sustainability indices using Ellipro (an online tool which predicts discontinuous epitopes from 3D structures proteins PDB format, based on solvent-accessibility and

flexibility; http://tools.immuneepitope.org/tools/ElliPro/iedb_input). The PDB format input files for HA2 were provided to the server separately and minimum score value was set at 0.7 while maximum distance was selected as $6 \AA$. A residue clustering algorithm, the MODELLER program, and the Jmol viewer, together with this tool allowed the prediction and visualization of epitopes of antibody in protein sequences and structures [19] allergenicity [20] and structure validity using PSVS V (Version 1.5, PDBStat Version 5.12) [21].

Isolation of the HA2 gene

The pGEM-T vector containing the complete HA gene of A/Tehran/18/2010/H1N1, isolated in Pasteur Institute of Iran, was used as a PCR template in this study. To facilitate the cloning procedure, Bam $\mathrm{H} 1$ and HindIII restriction sites were embedded in the forward and reverse primers used for the amplification, respectively. The primer sequences with underlined restriction sites are shown in Table 1.

Table 1. Primers designed to reproduce HA2

Table 1. Primers designed to reproduce HA2
\begin{tabular}{|c|c|}
\hline Primer & sequence \\
\hline Forward primer & 5 '-TCGGATCCTCTATTCAATCTAG-3' \\
\hline Reverse primer & 5'-CC $\underline{\text { AAGCTTAATACATATTCTACAC-3' }}$ \\
\hline
\end{tabular}

PCR was conducted using a mixture of $2.5 \mu \mathrm{M}$ Taq polymerase buffer, $0.75 \mu \mathrm{g} \mathrm{MgCl} 2$, dNTP $(10 \mathrm{mM}), 1 \mu \mathrm{M}$ primers and Taq polymerase enzyme (CinnaGen, Iran). The PCR product was evaluated by running on $1 \%$ agarose gel electrophoresis [22].

\section{Cloning of the amplified HA2 fragment}

In order to clone HA2 in pGEM-T Easy vector, the fragment was purified from agarose gel using Gel Extraction kit (Bioneer, USA). The target gene and the vector were ligated using T4 DNA ligase (ThermoScientific, Lithuania). Following $24 \mathrm{~h}$ of the ligation, the vectors were transformed into competent Top 10F' Escherichia coli and cultured on solid medium containing IPTG inducer, X-Gal reagent and ampicillin and tetracycline antibiotics. The blue-white colony screening method was used to select the plasmid-derived colonies and the plasmids were investigated after extraction for the presence or absence of the desired gene by enzyme digestion by the two specific enzymes (BamH1 and HindIII).

The double-digested HA2 amplicon was purified to be used as an insert by PCR purification kit (Roche, Germany). At the same time, the expression vector pET28a was double-digested by the same two enzymes and using the silicate column of plasmid extraction kit (Qiagen, Germany), the linear vector was ligated into the HA2 insert and stored at $4^{\circ} \mathrm{C}$ for 4 days. Competent E. coli Top10F' was transformed and cultured on agar plate containing kanamycin and tetracycline. The colonies were evaluated by colony-touch PCR and enzymatic digestion. Finally, a confirmed recombinant vector was sent to SinaClone (Iran) for sequencing.

\section{Expression and purification of HA2 protein}

In order to express the protein, the recombinant plasmid was transformed into E. coli strain BL21. The expression of HA2 protein was induced by $1 \mathrm{mM}$ IPTG at $\mathrm{OD}=0.6-0.8$ and incubation at $37^{\circ} \mathrm{C}(190 \mathrm{RPM})$ for $4 \mathrm{~h}$. To optimize the expression conditions, IPTG was tested at two concentrations of 0.2 and $0.5 \mathrm{mM}$, incubated at two temperatures of $37^{\circ} \mathrm{C}$ and $28^{\circ} \mathrm{C}$. The presence of protein was investigated by SDS-PAGE, based on Lammeli method [23] and detected by Coomassie staining. Western blot was used to confirm the identity of the recombinant proteinsusing nitrocellulose membrane and anti $6 \times$ his tag antibody (1/6000 Qiagen, Germany). Finally, the protein band was visualized using Diabimabenzidine (DAB, Sigma, UK) substrate.

In order to purify the recombinant protein, Protino ${ }^{\circledR} \mathrm{Ni}-\mathrm{TED}$ 1000 packed column (MN, Macherey-Nagel, Germany) was used, according to the manufacturer's instructions. The extracted lysate was taken on the column in several steps. The samples collected from different steps of the column purification were analyzed by SDS-PAGE. The purified protein was then desalted with G25 columns and dialyzed against a PBS buffer.

\section{RESULTS}

\section{Bioinformatics analysis outcome}


The results of preliminary bioinformatics studies such as the homology of HA2 fragments among different influenza viruses and their physicochemical properties by the available software on Expasy website provided information on HA2 recombinant protein, such as its molecular weights $(\mathrm{Mw})$ of approximately $28 \mathrm{kDa}$, approximate half-life of more than $10 \mathrm{~h}$ in E. coli, the stability index of about 32.91 (below 40 indicates the stability of the molecule), estimated pI (6.3), aliphatic index (74.65) and GRAVY(-0.494) (negative GRAVY value indicates that the protein is non-polar) which were used in the next steps to identify and validate the molecule.

The allergenicity of HA2 protein was evaluated using Structural Database of Allergenic Proteins (SDAP) and the results showed that the protein was not allergenic.
This protein was also evaluated using IEDB database for epitope mapping and the scores of any amino acid in the antigenicity of the HA2 molecule were evaluated as shown in Fig. 1.

As shown in Fig. 2, the best epitopic regions, predicted by IEDB (Ellipro) were fully available on the molecular structure of the three-dimensional structure (MODELLER program and Jmol viewer) and were predicted to be potentially capable of stimulating the immune system and producing antibodies. Furthermore, the quality of the molecular structure was evaluated by Protein Structure Validation Software (PSVS) and the results showed that the protein model is acceptable (Table 2).

\begin{tabular}{|c|c|c|c|c|c|c|}
\hline Ho. $\triangleq$ & Chain $\Rightarrow$ & Start $₹$ & End $\Rightarrow$ & Peptide & Number of residues $\stackrel{-}{-}$ & Score $\uparrow$ \\
\hline 1 & - & 240 & 268 & SLGAISFMMCSNGSLQCGICIKLAAA,ALEH & 29 & 0.789 \\
\hline 2 & - & 171 & 208 & EIGNGCFEFYHKCDNTCMESVKNGTYDYPKYSEEAKLN & 38 & 0.767 \\
\hline 3 & - & 1 & 39 & MGSSHHHHHHSSGLVPRGSHMASMTGGQQMGRGSSIQSR & 39 & 0.761 \\
\hline 4 & - & 211 & 219 & EIDGVKLES & 9 & 0.687 \\
\hline 5 & - & 63 & 72 & YHHQNEQGSG & 10 & 0.593 \\
\hline 6 & - & 118 & 123 & NLNKKV & 6 & 0.569 \\
\hline 7 & _ & 48 & 53 & FIEGGW & 6 & 0.529 \\
\hline
\end{tabular}

Fig. 1. Predicted linear epitopes by Ellipro
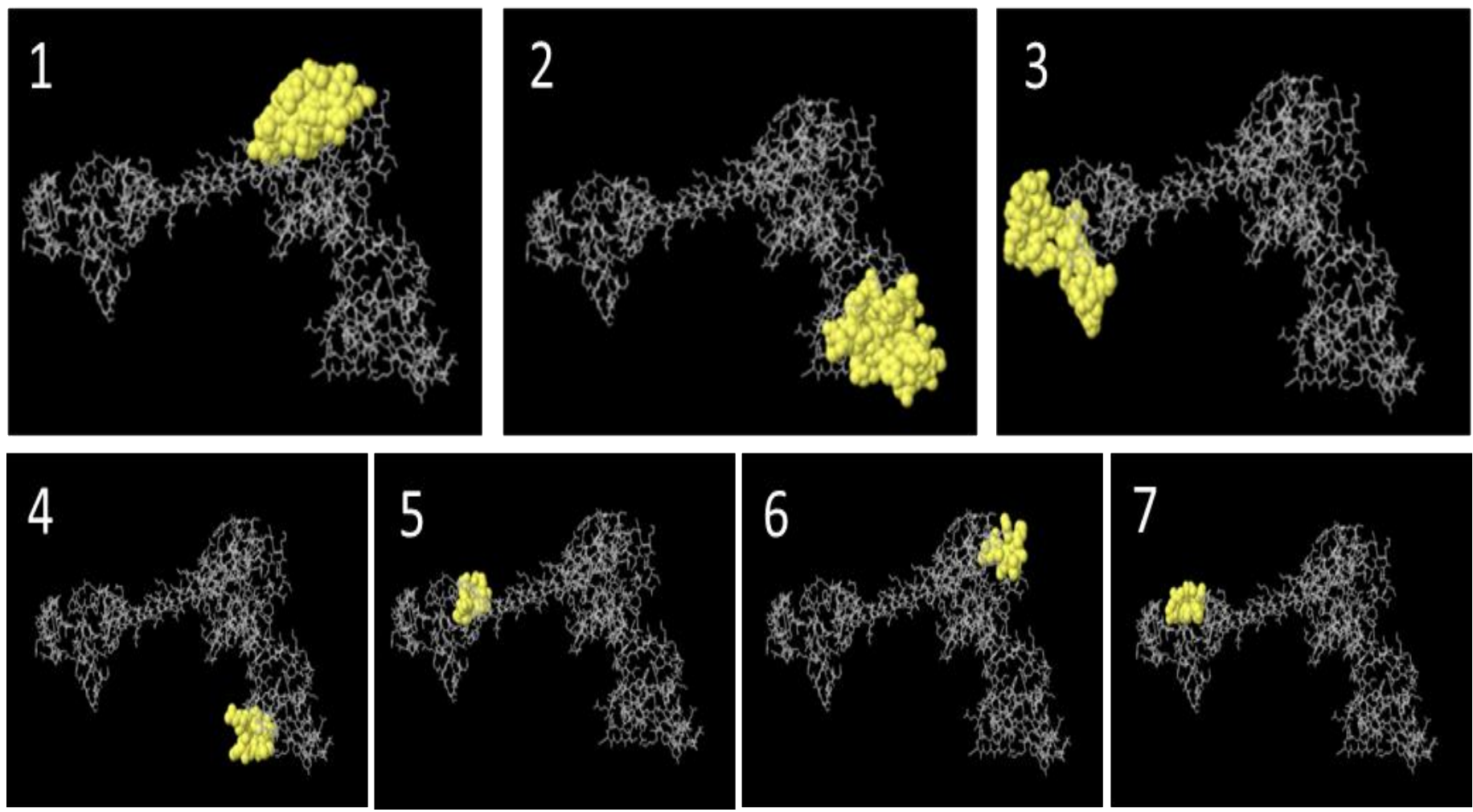

Fig. 2. 3D structure of $\mathrm{HA} 2$ molecule and important antigenic regions as determined by Ellipro. (In this ball-and-stick model, yellow balls are the residues of predicted epitopes and white sticks are the structures for the non-epitope and core residues) 
Table 2. Structural indices of HA2 protein using PSVS

\begin{tabular}{|c|c|c|c|c|c|}
\hline Program & Verify3D & ProsaII (-ve) & Procheck (phi-psi)3 & Procheck (all)3 & MolProbityClashscore \\
\hline Raw score & -0.17 & -0.06 & 0.27 & 0.10 & 92.59 \\
\hline Z-score1 & -4.65 & -2.94 & 1.38 & 0.59 & -14.36 \\
\hline
\end{tabular}

\section{HA2 isolation and cloning}

PCR amplification of the 700-bp HA2 amplicon using the designed primers is shown in Fig. 3.

The desired gene was successfully double-digested by Bam $\mathrm{H} 1$ and HindIII restriction enzymes from pGEM-T Easy vector for cloning in pET28a expression vector and was cloned into double-digested pET28a expression vector. Colonies harboring the vector following transformation were tested and confirmed by colony-touch PCR. The results, as expected, showed a 700bp amplicon for the positive colonies (Fig.4).

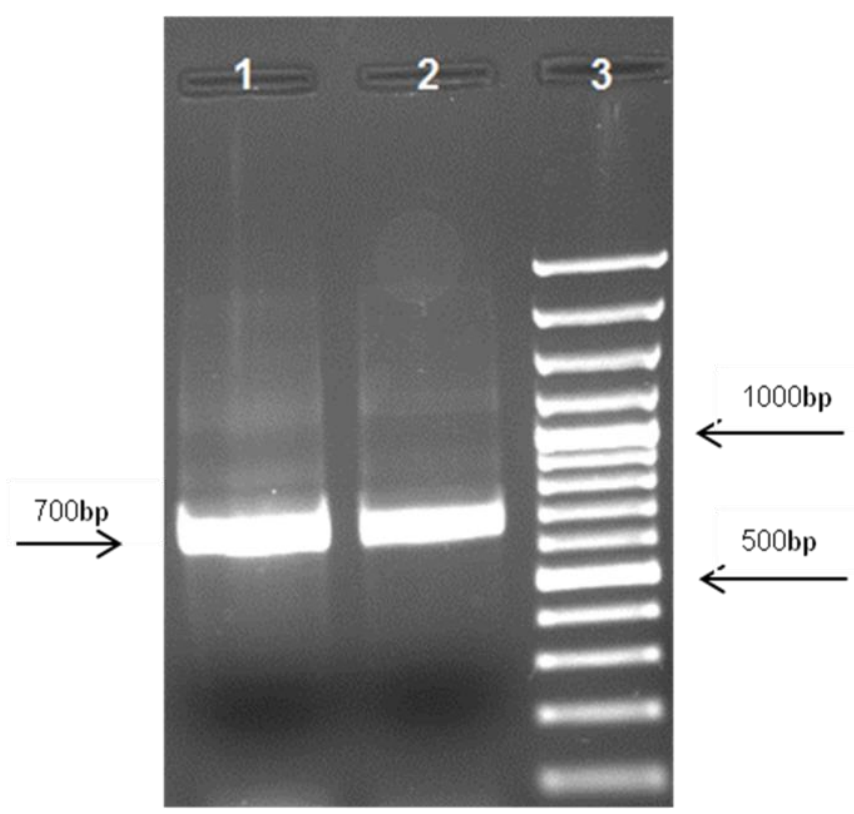

Fig. 3. PCR result of $\mathrm{HA} 2$ gene amplification on $1 \%$ agarose gel. Lanes 1 and 2: HA2 fragment, lane 3: DNA ladder (100-bp).
After the above-mentioned initial confirmation by PCR, the enzymatic digestion was carried out using Bam $\mathrm{H} 1$ and HindIII restriction enzymes on the extracted constructs of two positive colonies. The results confirmed the presence of a 700-bp (HA2) gene fragment (Fig. 5).

In order to confirm the sequence of the cloned gene, the recombinant pET28a /HA2 construct was sent for sequencing. The sequencing reviews confirmed the correctness of the cloned sequence and the absence of any mutations or false positives.

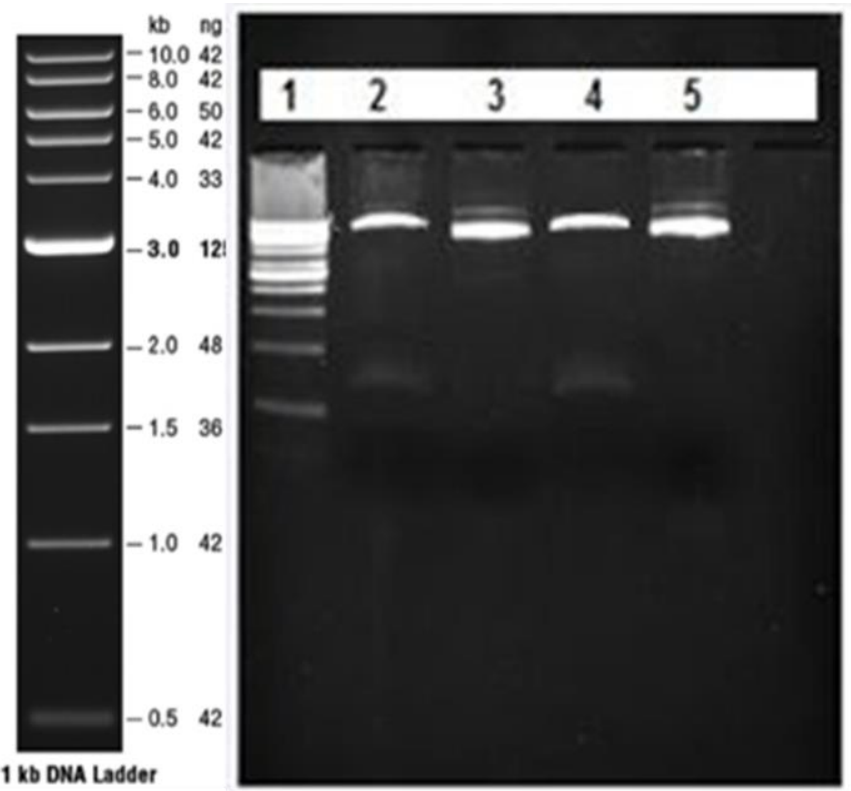

Fig. 5. The result of the enzymatic digestion of the recombinant construct. Lane 1: DNA ladder (1kbp). Lanes 2 and 4: The digested recombinant construct. Lanes 3 and 5: non-digested constructs, exhibiting super-coils.

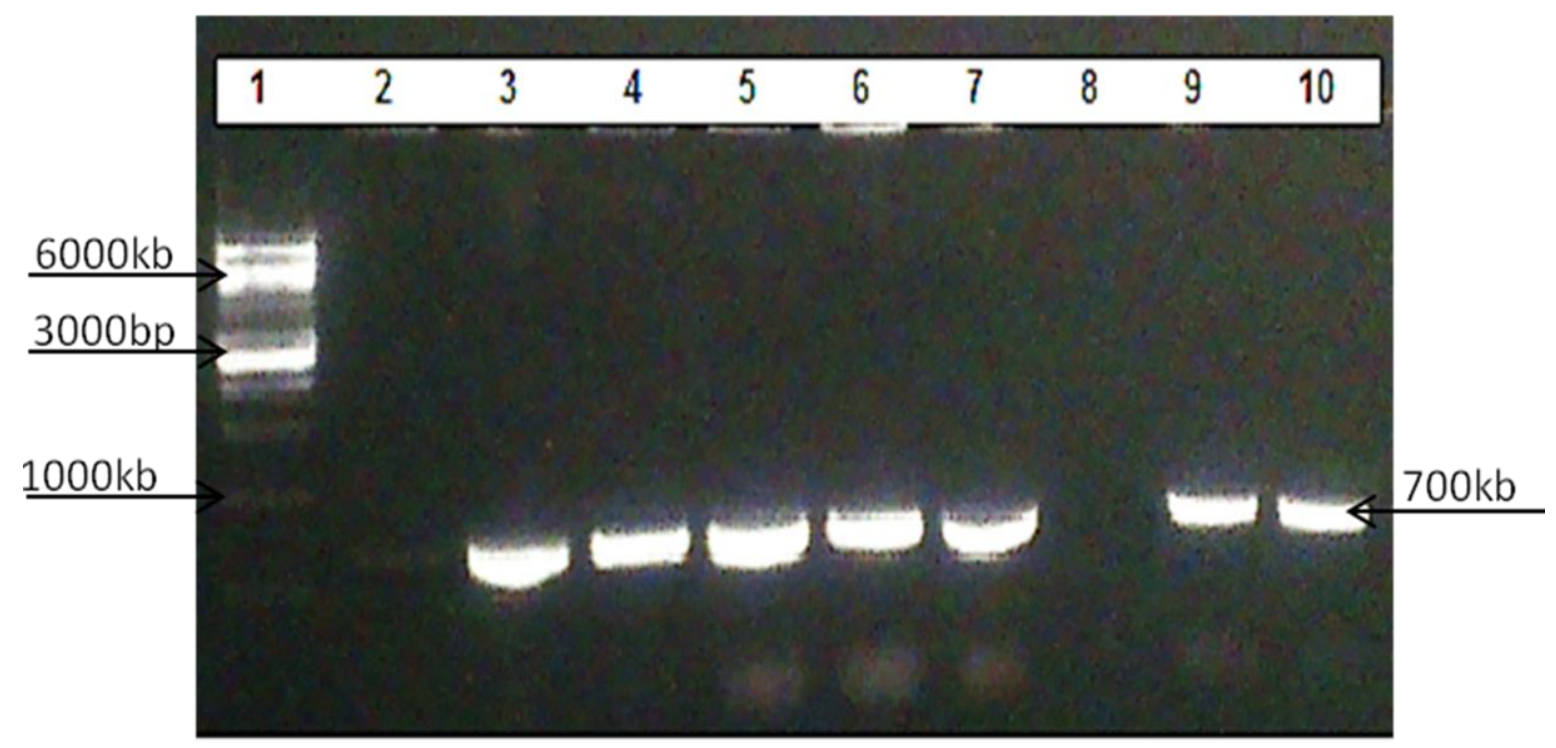

Fig. 4. Colony-touch PCR results. Lane 1: DNA ladder (1Kb), Lanes 2-10: conjugated clones. 


\section{Expression and purification of HA2 protein}

The confirmed recombinant construct was transformed into E. coli strain BL21 to express the HA2 protein. The protein expression was investigated with two concentrations of IPTG at different incubation times at $28^{\circ} \mathrm{C}$ and $37^{\circ} \mathrm{C}$. The results from SDS-PAGE showed that the highest and optimal expression induction condition was obtained at the concentration of 0.5 $\mathrm{mM}$ IPTG and $3 \mathrm{~h}$ after the induction, incubated at $28^{\circ} \mathrm{C}$ (Fig.

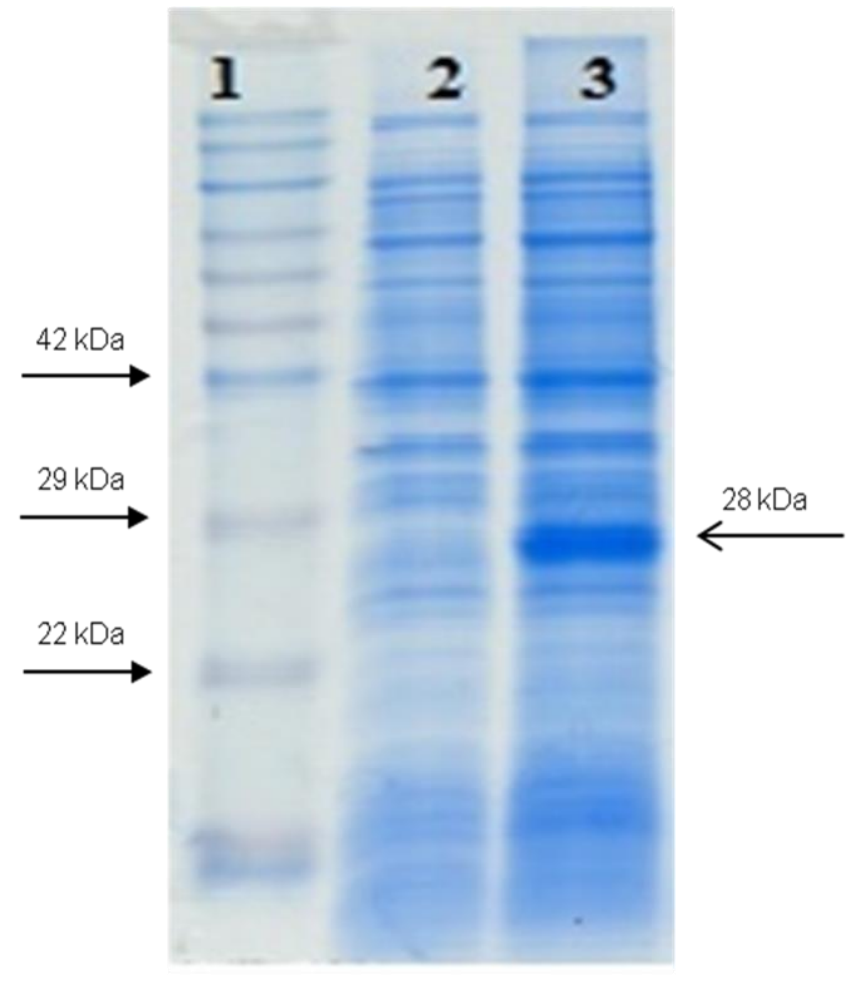

Fig. 6. SDS-PAGE result of HA2 protein expression at temperature of $28^{\circ} \mathrm{C}$. The bacterial lysis containing the recombinant plasmid pET28a /HA2. Lane 1: Protein marker, lane 2: Prior to induction with 0.5 IPTG (0 h), lane 3: After induction with $0.5 \mathrm{mM} \mathrm{IPTG}(3 \mathrm{~h})$

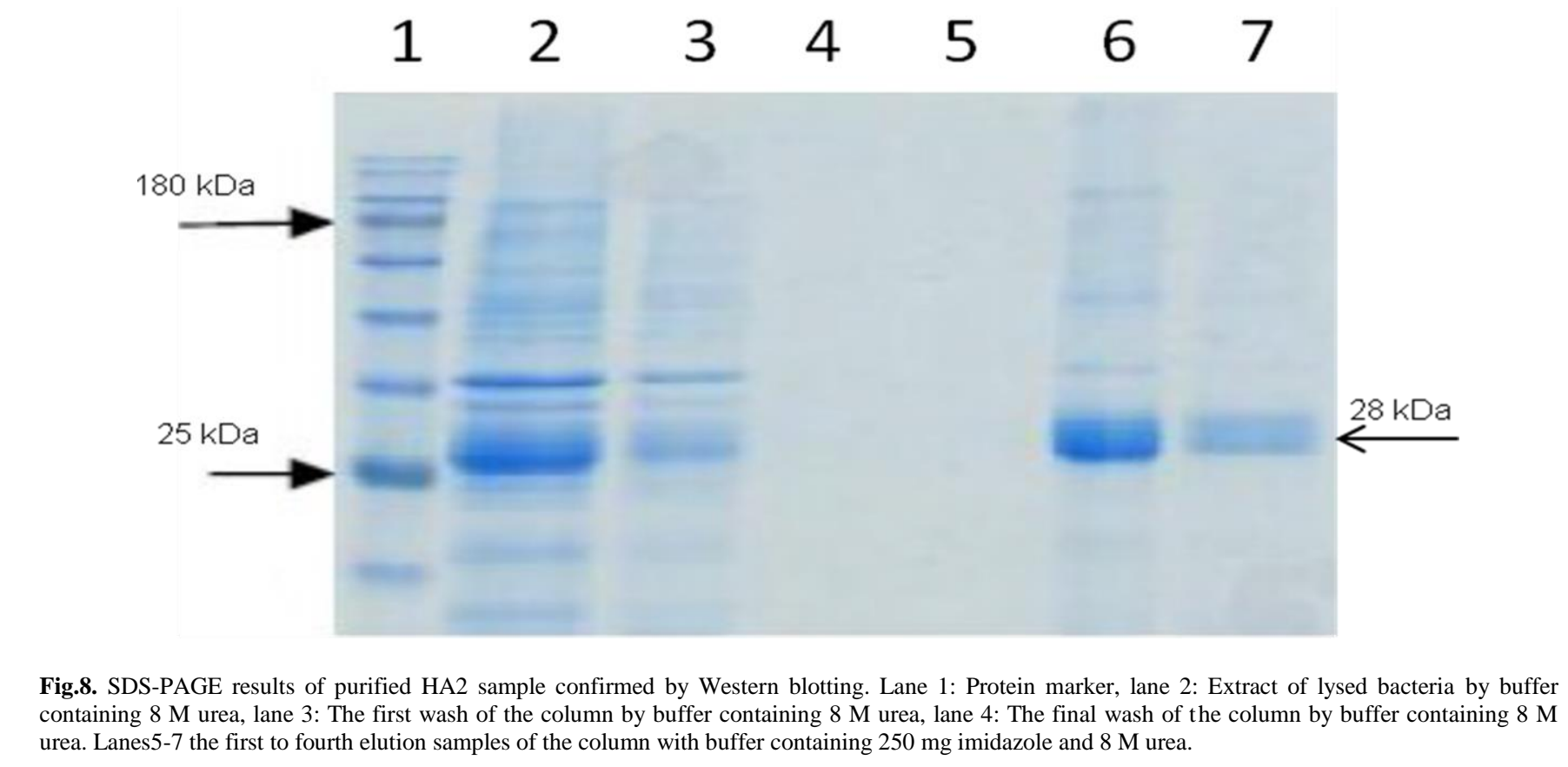

Fig.8. SDS-PAGE results of purified HA2 sample confirmed by Western blotting. Lane 1: Protein marker, lane 2: Extract of lysed bacteria by buffer containing $8 \mathrm{M}$ urea, lane 3: The first wash of the column by buffer containing $8 \mathrm{M}$ urea, lane 4 : The final wash of the column by buffer containing $8 \mathrm{M}$ urea. Lanes5-7 the first to fourth elution samples of the column with buffer containing $250 \mathrm{mg}$ imidazole and $8 \mathrm{M}$ urea.
6). The protein expression was also examined after one night. The result showed a significant decrease in protein expression.

The final confirmation of the recombinant His-tagged HA2 protein was carried out by Western Blotting. The obtained results as shown in Fig.6 indicated the detection of recombinant His-tagged HA2 protein band in the range of $28 \mathrm{kDa}$.

The observed results in Figs. 7 and 8 show the presence of HA2 protein band in the range of $28 \mathrm{kDa}$. The purified protein concentration was approximately $0.4 \mathrm{mg} / \mathrm{ml}$.

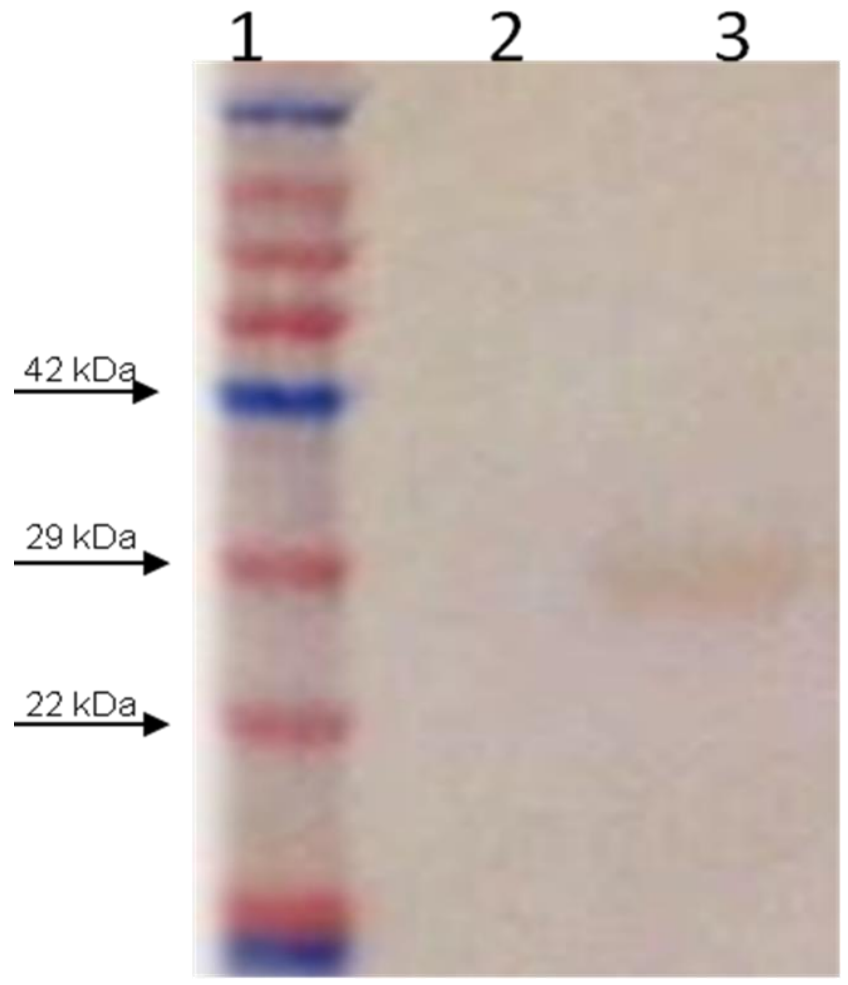

Fig.7. Western blot analysis of HA2 Protein. Lane 1: Protein marker, lane 2: Cell lysis before expression induction, lane 3: HA2 protein-expressing cell lysate at $3 \mathrm{~h}$ after induction with $0.5 \mathrm{mM}$ IPTG. 


\section{DISCUSSION}

Due to the high prevalence of influenza epidemics that occur every year, several studies are ongoing on its prevention and treatment. Vaccination is the most efficient and cost-effective way to prevent infectious diseases [24]. Inactivated flu vaccines have the benefit of preventing the disease, however, because of poor immune response in neonates and the elderly (over 65 years of age) and the constant need to follow changes in the viral proteins, especially $\mathrm{HA}$ and NA, the researchers have to look for more efficient vaccines that need no annual change but provide acceptable cross-linking immunity [25].

As stated, the most important challenge in the production of a subunit recombinant flu vaccine is the genetic instability of the virus proteins which limits the application of the vaccine in the long term [26]. For this reason, the use of conserved sequences in the viral antigenic proteins is preferable to develop the new vaccines. The results of the alignment of the influenza virus genes amongst different types of the virus indicated that HA gene is highly conserved in some areas of the molecule. Despite the high mutation rate in the large subunit of HA glycoprotein (HA1), its small subunit (HA2) is highly conserved. Due to the fact that HA2 sequence, especially in first 11 amino acids are conserved among the various subtypes of the virus, it can be inducer of acquired immunity for the different subtypes. Thus, antibodies targeting HA2 subunit will provide a widespread protection against the seasonal and pandemic infections of various types of influenza A viruses. This protection is provided through the prevention of HA2 integration activities. For this reason, HA2 is one of the most important antigens to produce a broad-spectrum vaccine. Studies have been conducted on the immuno-protective effects of the conserved areas of HA2 which all have shown, it could be considered as a remarkable imitated candidate for the infection [27, 28]

In case of HA1, A study has shown that the HA1 molecule of the influenza A virus/ H1N1/ 2009 expressed in E. coli in nonglycosylated form was completely immunogenic and stimulated the immune system in an animal model [29]. The antibodies produced against that antigen have been shown to have the ability to react with HA1 antigen and to exhibit favorable protective effects. It has been shown that due to genetic variations of the virus, especially in HA molecule, the position and number of glycan chains on this molecule are variable but have no effect on its spatial structure and molecular function [29]. In another study, it has been shown that the shorter the length of the glycan chains on HA molecule (even as monosaccharides), the stronger the antigenicity of the molecule will be [30].

In this study, considering the importance of HA2 conserved areas for the immune response and the lack of glycosylation effect on the structure of this peptide, we attempted to clone HA2 into a prokaryotic expression vector. Given the above information and the lower efficacy and protective effects of the inactivated available vaccines compared to subunit vaccines and their time-consuming production in face of the pandemic (e.g. in 2009 pandemic required 150 million doses for only Latin America only) [29], we cloned and expressed and detected this HA2-based vaccine candidate as a histidine-tagged recombinant protein. Following bioinformatics analyses of the target protein in terms of different indices as the first step, we observed that HA2 molecule could be expressed well in a prokaryotic host and based on the predicted epitopic sites, it can potentially stimulate the immune system to produce polyclonal antibodies against the virus and can be considered as a good candidate global vaccines to prevent emerging pandemics and epidemics. In order to use this component in the combination of an effective vaccine against influenza, evaluating the immunogenicity of the protein in animal models such as mice is essential. For instance, this protein can be evaluated by using an adjuvant or adding other influenza virus conserved proteins such as nucleoprotein (NP) or parts of type II membrane proteins $(3 \mathrm{M} 2 \mathrm{e})$. Furthermore, the production of monoclonal antibody based on this antigen can also be pursued.

\section{ACKNOWLEDGEMENT}

This work was funded by grant No 759, from Pasteur Institute of Iran.

\section{CONFLICT OF INTEREST}

The authors declare that they have no conflict of interest.

\section{REFERENCES}

1. Liu D, Si B, Li C, Mi Z, An X, Qin C et al. Prokaryotic expression and purification of HA1 and HA2 polypeptides for serological analysis of the 2009 pandemic H1N1 influenza virus. J Virol Methods. 2011;172(1-2):1621. doi:https://doi.org/10.1016/j.jviromet.2010.12.007.

2. Hovden AO. The effect of influenza virus vaccine formulation: University of Bergen; 2005.

3. Mittelholzer CM. Influenza Virus Protection and Adaptation: Stockholm; 2006.

4. Varečková E, Mucha V, Kostolanský F. HA2 glycopolypeptide of influenza A virus and antiviral immunity. Acta Virol. 2013;57(2):247-56.

5. Bommakanti G, Citron MP, Hepler RW, Callahan C, Heidecker GJ, Najar TA et al. Design of an HA2-based Escherichia coli expressed influenza immunogen that protects mice from pathogenic challenge. PNAS. 2010;107(31):13701-6. doi:10.1073/pnas.1007465107.

6. Thoennes S, Li ZN, Lee BJ, Langley WA, Skehel JJ, Russell RJ et al. Analysis of residues near the fusion peptide in the influenza hemagglutinin structure for roles in triggering membrane fusion. Virology. 2008;370(2):403-14.

7. Staneková Z, Varečková E. Conserved epitopes of influenza A virus inducing protective immunity and their prospects for universal vaccine development. Virol J. 2010;7:351-. doi:10.1186/1743-422X-7-351.

8. Gamblin SJ, Skehel JJ. Influenza hemagglutinin and neuraminidase membrane glycoproteins. J Biol Chem. 2010;285(37):28403-9. doi:10.1074/jbc.R110.129809.

9. Curtis-Fisk J, Spencer RM, Weliky DP. Isotopically labeled expression in E. coli, purification, and refolding of the full ectodomain of the influenza virus membrane fusion protein. Protein Expr Purif. 2008;61(2):212-9. doi:10.1016/j.pep.2008.06.009.

10. Bardiya N, Bae JH. Influenza vaccines: recent advances in production technologies. Appl Microbiol Biotechnol. 2005;67(3):299-305. doi:10.1007/s00253-004-1874-1.

11. Kang SM, Song JM, Quan FS, Compans RW. Influenza vaccines based on virus-like particles. Virus Res. 2009;143(2):140-6. doi:10.1016/j.virusres.2009.04.005

12. Kim JI, Lee I, Park S, Park MS. Surface glycoproteins determine the feature of the 2009 pandemic H1N1 virus. BMB Rep. 2012;45(11):653-8. doi:10.5483/BMBRep.2012.45.11.137.

13. Russell RJ, Kerry PS, Stevens DJ, Steinhauer DA, Martin SR, Gamblin $\mathrm{SJ}$ et al. Structure of influenza hemagglutinin in complex with an inhibitor of membrane fusion. PNAS. 2008;105(46):17736-41. doi:10.1073/pnas.0807142105.

14. Sui J, Hwang W, C., Perez S, Wei G, Aird D, Chen LM et al. Structural and functional bases for broad-spectrum neutralization of avian and human influenza A viruses. Nat Struct Mol Biol. 2009;16(3):265-73. doi:10.1038/nsmb.1566.

15. Kang SM, Song JM, Compans RW. Novel vaccines against influenza viruses. Virus Res. 2011;162(1-2):31-8. doi:10.1016/j.virusres.2011.09.037. 16. Fouchier RA, Munster V, Wallensten A, Bestebroer TM, Herfst S, Smith 
$\mathrm{D}$ et al. Characterization of a novel influenza A virus hemagglutinin subtype (H16) obtained from black-headed gulls. J Virol. 2005;79(5):2814-22.

17. Yousefi A, Fotouhi F, Hosseinzadeh S, Kheiri MT, Farahmand B, Montazeri $S$ et al. Expression of antigenic determinants of the haemagglutinin large subunit of novel influenza virus in insect cells. Folia Biol (Praha). 2012;58(4):151-6.

18. Gasteiger E, Gattiker A, Hoogland C, Ivanyi I, Appel RD, Bairoch A. ExPASy: the proteomics server for in-depth protein knowledge and analysis. Nucleic Acids Res. 2003;31(13):3784-8.

19. Ponomarenko J, Bui HH, Li W, Fusseder N, Bourne PE, Sette A et al. ElliPro: a new structure-based tool for the prediction of antibody epitopes. BMC Bioinformatics. 2008;9(1):514. doi:10.1186/1471-2105-9-514.

20. Ivanciuc O, Schein $\mathrm{CH}$, Braun W. SDAP: database and computational tools for allergenic proteins. Nucleic Acids Res. 2003;31(1):359-62.

21. Bagaria A, Jaravine V, Huang YJ, Montelione GT, Güntert P. Protein structure validation by generalized linear model root-mean-square deviation prediction. Protein Science : A Publication of the Protein Society. 2012;21(2):229-38. doi:10.1002/pro.2007.

22. Farahmand B, Khodabandeh M, Mahboudi F, Fotouhi F, Barkhordari F, Saleh $M$ et al. Isolation, cloning, and sequencing of influenza A (H1N1) hemagglutinin for production of hemagglutinin gene bank. AMUG. 2011;13(4):59-67.

23. Laemmli UK. Cleavage of structural proteins during the assembly of the head of bacteriophage T4. Nature. 1970;227(5259):680-5.
24. Greenwood B. The contribution of vaccination to global health: past, present and future. Philos Trans $\mathrm{R}$ Soc Lond $\mathrm{B}$ Biol sci. 2014;369(1645):20130433

25. Zhu W, Wang CC, Wang BZ. From variation of influenza viral proteins to vaccine development. Int J Mol Sci. 2017;18(7):1554 doi:10.3390/ijms 18071554

26. Mostafa A, Kanrai P, Petersen H, Ibrahim S, Rautenschlein S, Pleschka $\mathrm{S}$. Efficient generation of recombinant influenza A viruses employing a new approach to overcome the genetic instability of HA segments. PLoS One. 2015;10(1):e0116917. doi:10.1371/journal.pone.0116917.

27. Lee JS, Chowdhury MY, Moon HJ, Choi YK, Talactac MR, Kim J, H et al. The highly conserved HA2 protein of the influenza a virus induces a cross protective immune response. J Virol Methods. 2013;194(1):280-8. doi:https://doi.org/10.1016/j.jviromet.2013.08.022.

28. Steel J, Lowen AC, Wang TT, Yondola M, Gao Q, Haye K et al. Influenza virus vaccine based on the conserved Hemagglutinin stalk domain. mBio. 2010;1(1):e00018-10. doi:10.1128/mBio.00018-10.

29. Aguilar-Yáñez JM, Portillo-Lara R, Mendoza-Ochoa GI, GarcíaEchauri SA, López-Pacheco F, Bulnes-Abundis D et al. An influenza A/H1N1/2009 hemagglutinin vaccine produced in Escherichia coli. PLoS ONE. 2010;5(7):e11694. doi:10.1371/journal.pone.0011694.

30. Wang CC, Chen JR, Tseng YC, Hsu CH, Hung YF, Chen SW et al. Glycans on influenza hemagglutinin affect receptor binding and immune response. Proc Natl Acad Sci USA. 2009;106(43):18137-42. doi:10.1073/pnas.0909696106. 